\title{
ORIGINAL ARTICLE Use of screening to recruitment ratios as a tool for planning and implementing spinal cord injury rehabilitation research
}

\author{
BC Craven ${ }^{1,2}$, C Balioussis ${ }^{1}$, SL Hitzig ${ }^{1,3}$, C Moore ${ }^{1,4}$, MC Verrier ${ }^{1,5}$, LM Giangregorio ${ }^{1,6}$ and MR Popovic ${ }^{1,7}$
}

Study design: Descriptive report.

Objectives: To describe screening to recruitment $(\mathrm{S}: \mathrm{R})$ ratios and discuss their use for planning and implementing research among individuals with spinal cord injury (SCI).

Setting: Toronto, Ontario, Canada.

Methods: We calculated S:R ratios for $\mathrm{SCl}$ research by study methodology and nature of the exposure/intervention for 25 studies previously conducted in a tertiary SCI rehabilitation facility. Study methodologies included ten randomized controlled trials (RCTs), nine cohort studies and six panel studies. Exposures included seven rehabilitation interventions, and three drug studies, ten telephone interviews/chart abstractions (TI/CA) and five surveys. A S:R ratio was calculated for each study methodology, and exposure type, by dividing the number of consenting individuals who underwent screening by the number of eligible recruited participants enrolled in the study.

Results: In terms of design, RCTs had the highest median S:R ratio (3:1), followed by cohort studies (2:1) and panel studies (2:1). In terms of intervention type, drug studies had the largest median $S: R$ ratio (5:1), followed in descending order by rehabilitation studies (2:1), TI/CAs studies (2:1) and surveys (2:1).

Conclusions: Reported $S: R$ ratios varied substantially with study methodology and the associated study intervention exposure. Awareness of $S: R$ ratios may assist researchers in estimating recruitment timelines, personnel needs and study budgets for a required sample size based on the planned study methodology and intended study exposure. We advocate for the routine reporting of $\mathrm{S}: \mathrm{R}$ ratios to inform the success of future $\mathrm{SCl}$ research.

Spinal Cord (2014) 52, 764-768; doi:10.1038/sc.2014.126; published online 19 August 2014

\section{INTRODUCTION}

Traumatic spinal cord injury (SCI) results in diverse motor, sensory and autonomic impairments caused by some form of external physical impact (that is, fall, motor vehicle accident, and so on). The extent of impairment is determined by the anatomic location and severity of the injury to the spinal cord. In Canada, it is estimated that there are currently nearly 44000 people living with traumatic SCI. ${ }^{1}$ In the year 2010, there were 1785 new cases of traumatic SCI in Canada, ${ }^{1}$ with $\sim 1500$ new cases occurring every year. ${ }^{2}$ In Ontario, the average age-standardized incidence rate of SCI between the years 2003 and 2006 was 2.4 injuries per 100000 people, ${ }^{3}$ with an estimated Canadian SCI prevalence of $1 / 10000$, whereas the United States reports an annual SCI incidence rate of about four injuries per 100000 people. ${ }^{4}$

Based on this demographic information, traumatic SCI cannot be defined as a rare disease as conceptualized in the United States ${ }^{5}$ and Europe. ${ }^{6}$ Despite this, the recruiting challenges faced by researchers conducting SCI studies are similar to those faced in clinical research conducted among individuals with a rare disease, or one with low incidence and prevalence. In fact, finding eligible and willing research participants in the SCI population, and obtaining an adequate sample size, poses a unique set of challenges for researchers. ${ }^{7}$ One reason is that SCI is a very broad classification, and the population of individuals with SCI are heterogeneous with respect to many characteristics often used to identify multiple subgroups, including, but not limited to, the etiology of the injury, age at injury, the neurological level of injury and preservation of sublesional sensory function and motor ability. ${ }^{8}$ Obtaining eligible SCI participants from one of these subgroups may therefore require application of strict pathophysiological inclusion and/or exclusion criteria, thus further restricting the pool of potential SCI participants and prolonging the duration of the study. ${ }^{9}$ Prolongation of studies beyond the expected timeline may lead to draining of resources, and funding shortages associated with lack of recruitment progress. ${ }^{10}$ In an effort to avoid these issues, and boost recruitment, researchers may be tempted to amend inclusion/exclusion criteria midstudy; this practice, however, exposes the entire study to bias, and compromises the integrity of the results. Obtaining an adequate sample size is vital to the success and relevance of any study regardless of design or primary outcome of interest. ${ }^{11}$ This issue is not unique to SCI, $85 \%$ of clinical trials do not conclude on time due to inadequate participant recruitment and

${ }^{1}$ Toronto Rehabilitation Institute - University Health Network, Toronto, Ontario, Canada; ${ }^{2}$ Department of Medicine and Health Policy Management and Evaluation, University of Toronto, Toronto, Ontario, Canada; ${ }^{3}$ Faculty of Medicine, Institute for Life Course and Aging, University of Toronto, Toronto, Ontario, Canada; ${ }^{4}$ University of Waterloo, Waterloo, Ontario, Canada; ${ }^{5}$ Department of Physical Therapy, University of Toronto, Toronto, Ontario, Canada; ${ }^{6}$ Department of Kinesiology, University of Waterloo, Waterloo, Ontario, Canada and ${ }^{7}$ Rehabilitation Engineering Laboratory, Institute of Biomaterials and Biomedical Engineering, University of Toronto, Toronto, Ontario, Canada

Correspondence: Dr BC Craven, Toronto Rehabilitation Institute - University Health Network, Lyndhurst Centre, 520 Sutherland Drive, Toronto, Ontario, Canada M4G 3 9. E-mail: cathy.craven@uhn.ca

Received 8 January 2014; revised 20 May 2014; accepted 28 May 2014; published online 19 August 2014 
$60-80 \%$ of trials do not meet their temporal endpoint due to recruitment challenges. ${ }^{9}$ Insufficient sample size can decrease the ability of a study to evaluate treatment efficacy and decrease the chance of detecting statistically and/or clinically relevant interventions. ${ }^{11,12}$ Recruiting an insufficient number of participants also affects the internal and external validity of a study, inhibits the development of reliable evidence and delays adoption of effective interventions. Consequently, inability to attain an adequate number of participants can derail even the most meticulously designed, wellexecuted study.

To optimize the number of subjects/participants enrolled in a study and minimize time and expense, researchers are advised to lay out a recruitment strategy that includes a well-defined and justified screening procedures. ${ }^{13} \mathrm{~A}$ screening procedure identifies individuals who meet the inclusion criteria and do not meet exclusion criteria laid out in the study design. Robust screening will help formulate an effective study and guard against patient dropout midstudy. Designing an appropriate screening process is therefore vital to establishing eligible patient populations and suitably approaching patient sources. ${ }^{7}$ The screening process will ensure willing participants meet safety guidelines agreed upon by investigators and ethics boards, ${ }^{9}$ and provide opportunities for participating in the research for which they are eligible.

It is therefore paramount to develop the necessary tools to support the implementation of the screening procedure. Identifying the ratio of people that should be screened to successfully enroll one participant would provide researchers the ability to estimate the time and resources required for optimal subject accrual in a predictable timeframe. The process typically involves calculating the screening to recruitment (S:R) ratio (or recruitment rate), which is a method used for other health populations where low recruitment and/or high attrition hinder the conduct or completion of research trials (for example, pediatrics, ${ }^{14}$ surgery $\left.{ }^{15}\right)$. S:R ratios enable researchers to make informed estimates regarding the timeline, budget and personnel needs to successfully complete studies in specific research populations.
This paper describes and compares the S:R ratios of different SCI rehabilitation study methodologies (randomized controlled trials (RCTs), cohort and panel studies) and exposure/intervention types (rehabilitation, drug, telephone interview/chart abstraction, and survey) conducted at a Canadian tertiary rehabilitation facility for persons with SCI. The purpose of this report is to provide estimates of $\mathrm{S}: \mathrm{R}$ ratios for SCI research using a variety of designs, and discuss potential implications of the routine use of $S: R$ ratios as a tool for planning and implementing SCI rehabilitation research.

\section{MATERIALS AND METHODS}

Twenty-five studies conducted in or affiliated with a SCI tertiary rehabilitation center in Toronto, Ontario, Canada, were pooled. All studies had comprehensive prescreening, screening and recruitment data. Primary or site investigators were contacted and asked to share their screening procedure, number of individuals screened, the number of individuals enrolled, study design and sample size. Of the 32 studies identified among group members, twenty-five studies had adequate documentation of recruitment, prescreening and consent procedures, and were included in the study. For multicenter studies, only local site-specific data are reported. Only participants with SCI were included in the analysis; able-bodied controls or controls with similar impairments were omitted. The S:R ratios were calculated based on the number of consenting individuals who underwent screening divided by the number of eligible individuals enrolled in the study. All ratios are reported with a denominator of one (that is, the number of individuals screened to enroll one participant). The 25 studies comprise 10 RCTs, 9 cohort studies and 6 panel studies. RCTs consisted of rehabilitation and drug interventions. Cohort and panel studies included medical chart abstraction as well as telephone, mail and face-to-face interviews. The $\mathrm{S}: \mathrm{R}$ ratios for each study methodology and exposure/ intervention type were calculated and the median ratios and average sample sizes were reported.

\section{Study design}

There were three study methodologies identified: RCTs, cohort studies and panel studies. Table 1 provides the operational definitions for each of these study methodologies. Figures 1-3 provide schematic representations of the three study methodologies.

Table 1 Operational definitions for study methodologies and exposure/intervention types

\begin{abstract}
Study
methodology Description

RCTs Participants are randomly assigned to either a treatment or control group, and the outcome of the treatment is assessed and compared between groups. Include open, single-blind and double-blind study designs, depending on how much information is made available to participants. Results are quantitative and have the power to show cause-effect relationships between the intervention and the outcome (Figure 1 ). ${ }^{25}$

Cohort studies Examine individuals who are naturally divided into groups based on whether or not they possess a risk factor that is of interest, then followed over time and evaluated on the incidence of an outcome. ${ }^{26}$ Evaluation can take the form of surveys, interviews and/or chart abstraction, applied in a prospective or retrospective fashion. Compared with RCTs, cohort studies allow researchers to establish correlations over time and are less invasive to perform, but usually require more participants to achieve sufficient power to show relationships between variables. ${ }^{26}$ Cohort studies are also referred to as longitudinal, incidence or follow-up studies (Figure 2).

Panel studies Incorporate features of cross-sectional and cohort designs to evaluate changes in a sample at successive time intervals. ${ }^{27}$ Enable evaluation of temporal changes in health, lifestyle and quality of life using surveys, interviews, historical reviews, and so on. Unlike cohort studies, panel studies do not examine an intervention, but merely assess changes over time (Figure 3).
\end{abstract}

Exposure/intervention types

Rehab Examine types of therapy utilized in $\mathrm{SCl}$ rehabilitation (for example, functional electrical stimulation, whole body vibration, physical therapy, acupuncture, and so on).

Drug therapy Examine the effects of medications intended for individuals with $\mathrm{SCl}$.

(TI/CA) Incorporate one or more telephone interviews with medical information retrieved from a patient's medical record.

Survey Use standardized questionnaires to efficiently sample a population of people with $\mathrm{SCl}$.

Abbreviations: RCT, randomized controlled trial; rehab, rehabilitation; SCI, spinal cord injury; TI/CA, telephone interview/chart abstraction 


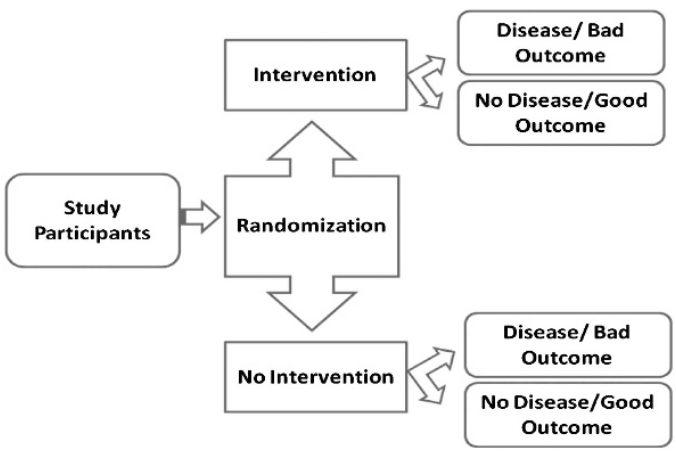

Figure 1 Schematic of RCT study methodology. ${ }^{23}$

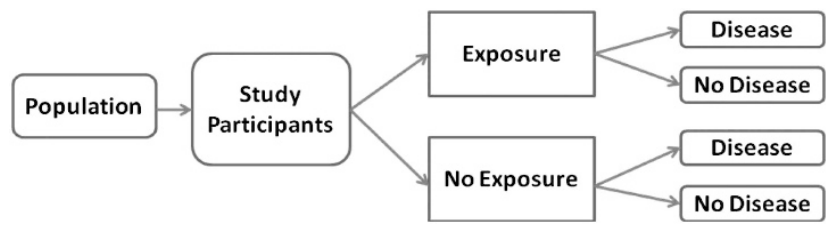

Figure 2 Schematic of cohort study methodology. ${ }^{24}$

\section{Exposure/intervention type}

Studies were classified into four exposure/intervention types: rehabilitation (rehab), drug therapy, telephone interview combined with chart abstraction (TI/CA) and survey. Table 1 presents the operational definitions for each of these intervention types.

\section{RESULTS}

Table 2 shows the design and intervention type, as well as the sample size and S:R ratio for each of the studies included.

\section{Study methodology}

Study methodologies included ten RCTs, nine cohort and six panel studies. Figure 4 shows that RCTs had the highest median S:R ratio, followed by cohort, and panel studies. Panel studies acquired the largest average sample size, whereas RCTs accrued the lowest average sample size.

\section{Exposure/intervention type}

There were seven rehab studies, three drug studies, ten TI/CAs and five surveys. As shown in Figure 5, drug studies had the largest median $S: R$ ratio, followed in decreasing ratios by rehab studies and TI/CAs. Surveys had the lowest median S:R ratio. TI/CA studies had the largest average sample size, followed by surveys, drug studies and rehab studies.

\section{DISCUSSION}

Participant recruitment is one of the most crucial and challenging factors in the success of a research study, ${ }^{9,10}$ and the present study represents a first effort to investigate $S: R$ ratios in different study types, for potential use in boosting participant recruitment. In the present study, we obtained a trend in the $S: R$ ratios for specific study methodologies or exposure/intervention types. Although these findings must be replicated in other settings, using larger sample sizes, the results of the present study suggest that $S: R$ ratios are a useful tool for estimating the number of participants that should be screened to meet enrollment goals. S:R ratios can be applied to the planning of budgets, timelines and personnel needs to successfully

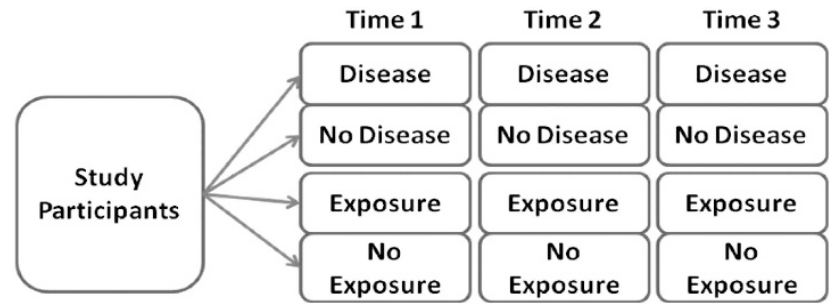

Figure 3 Schematic of panel study methodology.

complete recruitment of a desired sample size. S:R ratio data can also be used to assess the effectiveness of recruitment strategies and as a gauge of study validity.

The S:R ratios in the SCI studies reviewed varied by study methodology and exposure/intervention type. In terms of study methodology, RCTs in drug and rehabilitation therapy required the highest median number of people to be screened to enroll a single participant. Correspondingly, RCTs had much smaller sample sizes compared with the other two design types (panel and cohort), reflecting the relatively greater invasiveness and time commitment involved in RCT procedures, as well as the more rigorous inclusion/ exclusion criteria and validation process used.

In terms of exposure/intervention type, median $S: R$ ratios were lowest for surveys. Phone interviews do not require travel to an institution, facilitating ease of participation and larger sample sizes. However, results from survey studies can often be difficult to interpret and are unable to show cause-effect relationships. Drug studies had the highest median $\mathrm{S}: \mathrm{R}$ ratio, likely reflecting their greater perceived invasiveness and potential risks, compared with other study types. Although rehabilitation therapies do not have the stigma attached to drug therapy, they often require a substantial commitment of time and effort, perhaps explaining why rehab studies had the second highest median $S: R$ ratio.

$S: R$ ratios can be used to gauge how effectively researchers sample a study population, thus indicating external validity. ${ }^{16}$ Furthermore, obtaining $S: R$ ratio estimates before initiation of a study increases the credibility of the study's recruitment protocol by providing quantitative data to support estimations of the time, funding and resources needed to attain the desired sample size, based on study design and intervention type. These data can be included in proposals to research ethics boards, granting agencies and oversight organizations to improve the chances of study success. In addition, examining historical $S: R$ values for studies of similar methodology and exposure/intervention type can provide valuable insight into recruitment pitfalls, as well as a reference by which to gauge current recruitment success. ${ }^{17}$

Recruitment effectiveness can be hindered by several factors, ranging from trepidation toward a new drug or experimental treatment, ${ }^{18}$ to the method of screening. ${ }^{7}$ These types of issues become even more important for persons with catastrophic conditions, such as SCI. Preservation of a study's external validity through sufficient inclusion/exclusion criteria, imposed a priori, may assist in preserving $\mathrm{S}: \mathrm{R}$ ratios, particularly in a population as heterogeneous as that of persons with SCI.

One means by which researchers have attempted to improve recruitment in clinical trials is through central recruitment (CR), a process which recognizes that multiple uncoordinated recruitment attempts burden patients with SCI. CR involves having a single recruiter introduce multiple ongoing studies to each new patient. ${ }^{19}$ The CR process begins with an initial suitability assessment and 
Table 2 S:R ratio and sample size for all included studies, based on study exposure/intervention and methodology type

\begin{tabular}{|c|c|c|c|c|}
\hline Study name & Exposure/ intervention type & Study methodology & Sample size & $S: R$ ratio \\
\hline FES—walking-NCT 00201968 & Rehab & RCT & 22 & $3: 1$ \\
\hline ONBO-99145 & Rehab & $\mathrm{RCT}$ & 34 & $5: 1$ \\
\hline FES Grasp incompletes-NCT 00221117 & Rehab & $\mathrm{RCT}$ & 21 & 2.4:1 \\
\hline FES voluntary grasp incompletes-chronic NCT01208688 & Rehab & RCT & 8 & 7.3:1 \\
\hline ONRO-79 & Drug & $\mathrm{RCT}$ & 35 & $5.7: 1$ \\
\hline \multicolumn{5}{|l|}{ NCT 00150696} \\
\hline SCl-301+302 & Drug & $\mathrm{RCT}$ & 66 & $9.4: 1$ \\
\hline Bisphosphonate & $\mathrm{TI} / \mathrm{CA}$ & Cohort & 66 & $2.2: 1$ \\
\hline Calcium & $\mathrm{TI} / \mathrm{CA}$ & Cohort & 87 & $2: 1$ \\
\hline Bone cohort & $\mathrm{TI} / \mathrm{CA}$ & Cohort & 45 & $5.6: 1$ \\
\hline RHSCIR data ${ }^{a}$ & $\mathrm{TI} / \mathrm{CA}$ & Cohort & 185 & $1.7: 1$ \\
\hline SHAPE-SCI & $\mathrm{TI} / \mathrm{CA}$ & Panel & 334 & $3.8: 1$ \\
\hline Jousse-LTF & $\mathrm{TI} / \mathrm{CA}$ & Panel & 781 & $1.2: 1$ \\
\hline $\mathrm{SCl}$ adjustment & Survey & Panel & 50 & $5: 1$ \\
\hline SCIM & Survey & Panel & 35 & $1.9: 1$ \\
\hline TRI-01 & Survey & Panel & 59 & $1.5: 1$ \\
\hline VCUS-01 & Survey & Panel & 87 & $1.2: 1$ \\
\hline Autonomic dysreflexia & Survey & Cohort & 100 & $4.5: 1$ \\
\hline
\end{tabular}

Abbreviations: CR, central recruitment; FES, functional electrical stimulation; LTF, long term follow-up; RCT, randomized controlled trial; rehab, rehabilitation; RHSCIR, Rick Hansen Spinal Cord Injury Registry; SCI, spinal cord injury; SCIM, Spinal Cord Independence Measure; TI/CA, telephone interview/chart abstraction.

alndicates that CR was used in the study.

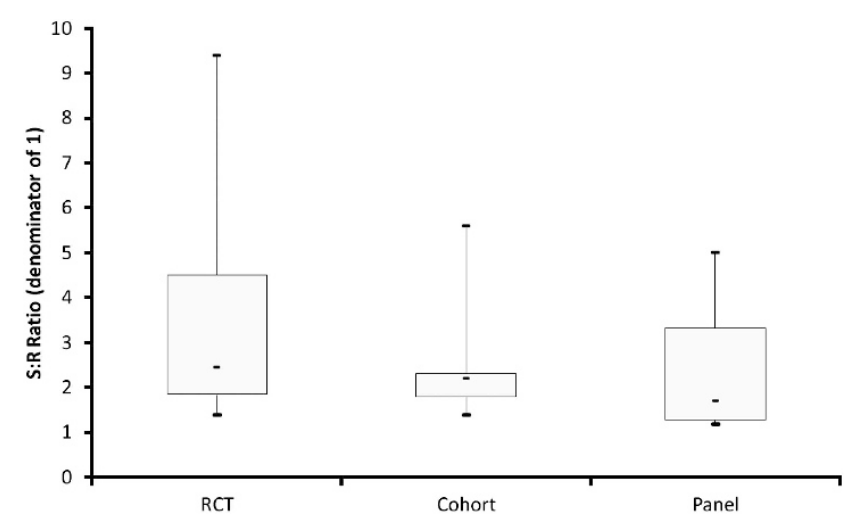

Figure 4 Box plots showing median, quartiles and range of $S: R$ ratios by study methodology. Median sample sizes were 34 (range 8-66) for RCT, 84 (range 41-395) for cohort and 73 (range 35-781) for panel studies.

health record screening (with the patient's consent). If eligible to participate in research, patients are approached by a research coordinator, provided with an inventory of available research studies, and informed of their eligibility for each relevant study. This allows patients to decide on their interest in, and availability for, participating in all current studies. ${ }^{19}$ The CR process aims to minimize patient burden, while providing individuals with all the

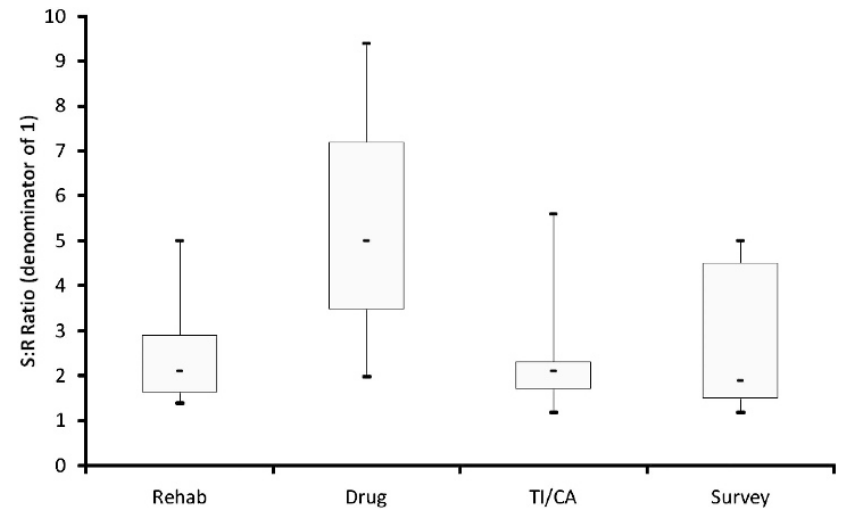

Figure 5 Box plots showing median, quartiles and range of $S: R$ Ratios by exposure/intervention type. Median sample sizes were 22 (range 8-47) for rehab, 40 (range 35-66) for drug, 85.5 (range 41-781) for telephone interviews/chart abstractions (TI/CA) and 59 (range 35-100) for survey studies.

information necessary for them to make informed decisions with regard to their potential participation in current available research studies.

The CR process is currently being pilot tested at Toronto Rehabilitation Institute - University Health Network's Lyndhurst Centre. Preliminary results have shown that: (a) the majority of patients $(80 \%)$ were interested in being approached about research, 
(b) the duration of time from patient admission to consent has improved by $30 \%$ and (c) the length of time from the first CR visit to informed consent decision was reduced in our site from 5.6 to 2.6 days in the first 6 months, representing a 50\% improvement. ${ }^{20}$ The CR process therefore appears to reduce the logistical and ethical challenges associated with recruiting subacute SCI inpatients to participate in research. The process has also been associated with high recruitment rates and a smoother, more efficient recruitment procedure. ${ }^{20}$ These characteristics make CR a promising recruitment process for SCI research, and further evaluation of the CR process scalability at Lyndhurst Centre is planned.

Limitations of the present study include that the data provided are site-specific, and that significance testing was not conducted, thus purely providing data trends. These factors limit the generalizability of the present study. In addition, a wide variety of prescreening techniques were employed (that is, referrals from medical professionals, word of mouth, poster campaigns, extensive telephone prescreening by a third party vendor, and so on.), which were not accounted for or reported in the present study and represent a substantial bias in the data reported. A standardized site-specific study screening procedure could reduce this limitation and provide valuable insight into the factors that prevent persons with SCI from participating in future research studies. The relatively small number of study designs described and intervention types evaluated was another limitation of the present study, in terms of generalizability. It is also noteworthy, however, that this analysis was specific to studies in the post-acute or rehabilitation stage, and not in the acute stage (for example, spinal cord repair or neuroprotection projects).

Despite these limitations, this paper marks the first reporting of multiple S:R ratios in SCI rehabilitation studies to date. To further address these issues, future investigations should examine a wider range of studies, with samples obtained from differing locations and conduct significance testing. In addition, future studies should look into quantifying the reasons for which individuals fail screening or do not agree to informed consent. Furthermore, routine reporting of $S: R$ ratios is advocated for all studies, ${ }^{16,21}$ to enable accurate tracking of the flow of participants throughout the course of the study, and future study comparisons, as well as inform future study designs. Currently, information on S:R ratios is often omitted from study results. ${ }^{16}$ The practices outlined above would shed light onto sources of patient recruitment, some flaws in research designs and point out aspects of selection bias inadvertently accounted for when constructing study protocols. New recruitment methods, such as electronic screening, ${ }^{22}$ may further assist in the provision of individualized screening for participants.

\section{DATA ARCHIVING}

There were no data to deposit.

\section{CONFLICT OF INTEREST}

The authors declare no conflict of interest.

\section{ACKNOWLEDGEMENTS}

This study was supported by the Ontario Neurotrauma Foundation (ONF Central Recruitment Grant \#2011-SCI-Mentor-884). We acknowledge the support of the Toronto Rehabilitation Institute, which received funding under the Provincial Rehabilitation Research Program from the Ministry of Health and Long-Term Care in Ontario during the conduct of many of the studies whose data are presented. The views expressed do not necessarily reflect those of the Ministry. We wish to thank the participants in all included studies. Due to site-specific reporting, sample sizes may differ to those obtained from the full study results. L. Giangregorio received an Early Researcher Award from the Ministry of Research and Innovation, and a Canadian Institutes of Health Research New Investigator Award.

1 Farry A, Baxter D. The incidence and prevalence of spinal cord injury in Canada. Overview and estimates based on current evidence. Rick Hansen Institute and Urban Futures: Vancouver, BC. 2010. Available from. http://fecst.inesss.qc.ca/filead min/documents/photos/LincidenceetlaprevalencedestraumamedullaireauCanada.pdf. (accessed 29 October 2013).

2 Ontario Neurotrauma Foundation. Spinal cord injury. Available from. http://onf.org/. (accessed 9 May 2013).

3 Couris CM, Guilcher SJ, Munce SE, Fung K, Craven BC, Verrier M et al. Characteristics of adults with incident traumatic spinal cord injury in Ontario, Canada. Spinal Cord 2010; 48: 45-50.

4 National Spinal Cord Injury Statistical Center. Spinal Cord Injury Facts and Figures at a Glance. Birmingham, AL, USA, 2012.

5 U.S. Government Printing Office. United States Public Law 107-280. Rare Diseases Act of 2002. U.S. GPO: Washington, DC, USA, 2002.

6 The Council of the European Union. Council recommendation of 8 June 2009 on an action in the field of rare diseases. Off J Eur Union 2009; 151: 7-10.

7 Cardenas D, Yilmaz B. Recruitment of spinal cord injury patients to clinical trials: challenges and solutions. Top Spinal Cord Inj Rehabil 2006; 11: 12-23.

8 Campagnolo D, Kirshblum S. Spinal Cord Medicine, 2nd edn, Lippincott Williams \& Wilkins: Philadelphia, PA, USA, 2011

9 Blanton S, Morris DM, Rettyman MG, McCulloch K, Redmond S, Light KE et al. Lessons learned in participant recruitment and retention: the EXCITE trial. Phys Ther 2006; 86: 1520-1533.

10 Bell KR, Hammond F, Hart T, Bickett AK, Temkin NR, Dikmen S. Participant recruitment and retention in rehabilitation research. Am J Phys Med Rehabil 2008; 87: 330-338.

11 Scales DC, Rubenfeld GD. Estimating sample size in critical care clinical trials. J Crit Care 2005; 20: 6-11.

12 Jadad AR, Moore RA, Carroll D, Jenkinson C, Reynolds DJ, Gavaghan DJ et al. Assessing the quality of reports of randomized clinical trials: is blinding necessary? Control Clin Trials 1996; 17: 1-12.

13 Aitken L, Gallagher R, Madronio C. Principles of recruitment and retention in clinical trials. Int J Nurs Pract 2003; 9: 338-346.

14 Saldaña SN, Hooper DK, Froehlich TE, Campbell KM, Prows CA, Sadhasivam S et al. Characteristics of successful recruitment in prospective pediatric pharmacogenetic studies. Clin Ther 2011; 33: 2072-2081.

15 Kaur G, Hutchison I, Mehanna H, Williamson P, Shaw R, Tudur Smith C. Barriers to recruitment for surgical trials in head and neck oncology: a survey of trial investigators. BMJ Open 2013; 3: 4

16 Schulz KF, Altman DG, Moher Dfor the CONSORT Group. CONSORT 2010 Statement updated guidelines for reporting parallel group randomized trials. BMJ 2010; 340: 698-702.

17 Jones LAT, Lammertse DP, Charlifue SB, Kirshblum SC, Apple DF, Ragnarsson KT et al. A phase 2 autologous cellular therapy trial in patients with acute, complete spinal cord injury: pragmatics, recruitment, and demographics. Spinal Cord 2010; 48: 798-807.

18 Hemminki E, Hovi SL, Veerus P, Sevon T, Tuimala R, Rahu M et al. Blinding decreased recruitment in a prevention trial of postmenopausal hormone therapy. J Clin Epidemio 2004; 57: 1237-1243.

19 Verrier M, Carson JR, Brisbois L, Craven BC. Central Recruitment Project: Exploring feasibility and scalability for research studies in spinal cord injury. Poster presented at: Translating Neural Engineering and Novel Therapies, 5th National Spinal Cord Injury Conference; 2012 October 19-20; Toronto, Ontario, Canada.

20 Craven BC, Brisbois LM, Verrier MC. Exploring the feasibility of central recruitment for subacute $\mathrm{SCl}$ patients. Poster presented at: Toronto Rehabilitation Institute Annual Research Day Conference; 2012 November 23; Toronto, Ontario, Canada.

21 Von Elm E, Altman DG, Egger M, Pocock SJ, Gøtzsche PC, Vandernbroucke JP. STROBE Initiative. The Strengthening the Reporting of Observational Studies in Epidemiology (STROBE) statement: guidelines for reporting observational studies. Epidemiology 2007; 18: 800-804.

22 Leveille SG, Huang A, Tsai SB, Weingart SN, Iezzoni LI. Screening for chronic conditions using a patient internet portal: recruitment for an internet-based primary care intervention. J Gen Intern Med 2008; 23: 472-475.

23 Streiner DL, Norman GR. PDQ Epidemiology, end edn. BC Decker, Inc: Hamilton, London, 1998.

24 Streiner DL, Norman GR. PDQ Epidemiology, end edn BC Decker, Inc: Hamilton, London, 1998, pp 68.

25 Sibbald B, Roland M. Understanding controlled trials: why are randomized controlled trials important? BMJ 1998; 316: 201.

26 Fletcher RW, Fletcher SW. Clinical Epidemiology: The essentials. Lippincott Williams \& Wilkins: Philadelphia, PA, USA, 2005

27 Oleckno WA. Epidemiology. Concepts and Methods. Waveland Press, Inc: Long Grove, IL, USA, 2008 